\title{
Involvement of prolactin and somatostatin in depression and the mechanism of action of antidepressant drugs
}

\author{
Agata Faron-Górecka, Maciej Kuśmider, Joanna Solich, \\ Magdalena Kolasa, Kinga Szafran, Dariusz Żurawek, Paulina Pabian, \\ Marta Dziedzicka-Wasylewska
}

Department of Pharmacology, Institute of Pharmacology, Polish Academy of Sciences, Smętna 12, PL 31-343 Kraków, Poland

Correspondence: Agata Faron-Górecka, e-mail:_gorecka@if-pan.krakow.pl

\begin{abstract}
:
Neuropeptides have been implicated in the physiology and pathophysiology of stress responses and therefore may play an important role in the pathogenesis of affective disorders such as Major Depression Disorder (MDD). The data presented in this mini-review demonstrate the role of prolactin (PRL) and somatostatin (STT) in the pathology and pharmacotherapy of MDD, focusing particularly on the response to antidepressant treatment, and compare the available data with the results obtained in our laboratory using the well-validated chronic mild stress (CMS) animal model of MDD.

Despite the availability of many pharmacological therapies for depression, ca. 35\% patients remain treatment resistant. This clinical situation is also true for rats subjected to CMS; some animals do not respond to antidepressant therapy and are considered treatment resistant. The most interesting results presented in this mini-review concern the changes in PRL and SST receptors in the brains of rats subjected to the full CMS procedure and IMI treatment and demonstrate the role of these receptors in the mechanisms of antidepressant action.

The possible interaction between SST and PRL, the involvement of the $\mathrm{D}_{2}$ dopamine receptor, and their direct protein-protein interactions are also discussed, with the conclusion that these two neurohormones play an important role in the mechanism of resilience after stress as well as in the mechanism of action of antidepressant drugs.
\end{abstract}

Key words:

prolactin, somatostatin, chronic mild stress model, treatment-resistant depression

\begin{abstract}
Abbreviations: $\left[{ }^{125} \mathrm{I}\right] \mathrm{PRL}-\left[{ }^{125} \mathrm{I}\right]$ prolactin, ADs - antidepressant drugs, BDNF - brain-derived neurotrophic factor, BIM-23A760 - chimeric somatostatin-dopamine compound, CMS - chronic mild stress, CNS - central nervous system, CSF - cerebrospinal fluid, DA - dopamine, $\mathrm{D}_{2} \mathrm{R}$ - dopamine receptor subtype 2 , GABA - $\gamma$-aminobutyric acid, GH growth hormone, HPA - hypothalamus-pituitary-adrenal, IMI - imipramine, MAO-I - monoamine oxidase inhibitors, MDD - major depression disorder, mRNA - messenger ribonucleic acid, NPY - neuropeptide Y, PRF - prolactin response to
\end{abstract}

fenfluramine, PRL - prolactin, PRLR - prolactin receptor, PRLR $_{\text {Long }}$ - long form of prolactin receptor, SSRI - selective serotonin reuptake inhibitors, stress NR - stress non-reactive animals, SST - somatostatin, SST-14 - somatostatin 14 form, SST-28 - somatostatin 28 form, sst $_{1} \mathrm{R}$ - somatostatin receptor type $1, \mathrm{sst}_{2} \mathrm{R}$ - somatostatin receptor type $2, \mathrm{sst}_{4} \mathrm{R}$ - somatostatin receptor type $4, \mathrm{sst}_{5} \mathrm{R}$ - somatostatin receptor type 5, stress- $\mathrm{R}$ - animals reactive to stress, TIDA - tuberoinfundibular dopamine pathway, TRP - L-tryptophan, YLD - years lived with disability 


\section{Introduction}

Depression is one of the most frequently occurring psychiatric diseases and a major cause of retraction from social life, measured as Years Lived with Disability (YLD), as well as premature death. The World Health Organization presents data indicating that depression is currently counted as the fourth most important world health problem. In both currently available diagnostic systems, depression is classified among the affective disorders. The actual causes of depression are not currently known. Major depression belongs to a complex group of disorders that manifest diverse clinical symptoms present to varying extents. The following symptoms are characteristic of the disorder: low mood, anhedonia, alterations in body weight not connected with diet, insomnia or hypersomnia, excitement or low motor activity, fatigue, feelings of guilt or/and worthlessness, lower intellectual abilities, lack of concentration as well as an inability to make decisions, and recurrent suicidal thoughts. Notably, major depression episodes occur twice as frequently in women $(20-25 \%)$ as in men (7-12\%) [17]. Women suffering from major depression are more frequently hospitalized, and their disorder is more likely to be chronic. Until now, there has not been a satisfactory explanation of this phenomenon, although depression more frequently occurs in women in the reproductive phase of life. Sex hormones may partly account for the higher risk of women developing depression, and transitions in the sex hormone levels in females (e.g., menarche, postpartum period, and menopause) are associated with increased vulnerability to depression [5].

The main environmental factor inducing depression is stress, which - at the neurochemical level manifests as hyperactivity of the hypothalamuspituitary-adrenal (HPA) axis. More than half of patients suffering from depression show hyperactivity of the HPA axis, which is resistant to feedback regulation (lack of dexamethasone inhibition). Despite the availability of many pharmacological and nonpharmacological therapies, ca. 35\% patients remain treatment resistant [24]. In addition, effective therapy leads to the normalization of HPA hyperactivity; antidepressant drugs (ADs) have been shown to inhibit HPA activity in human and animal studies [2] (Tab. 1). Episodes of depression most frequently occur with various stressogenic factors.

\section{Prolactin}

A frequent side effect of antidepressant therapy is hyperprolactinemia, but little data concerning these effects are available [4]. Hyperprolactinemia, usually defined as fasting levels of prolactin (PRL) at least $2 \mathrm{~h}$ after waking, is considered to be prolactin levels greater than $20 \mathrm{ng} / \mathrm{ml}$ in men and $25 \mathrm{ng} / \mathrm{ml}$ in women and is one of the most common endocrine dysfunctions of the HPA axis. Above all, drug-induced hyperprolactinemia after antipsychotic treatment is well documented, but the occurrence of this phenomenon after antidepressant treatment is less well known, although it has occasionally been reported with several classes of drugs. ADs with serotoninergic activity, including selective serotonin reuptake inhibitors (SSRI), can cause hyperprolactinemia through the enhancement of serotonin activity by inhibiting neuronal serotonin reuptake. Monoamine oxidase inhibitors (MAO-I) and some tricyclics also can raise prolactin levels by reducing catecholamines in the hypothalamus (Tab. 1). Moreover, some of the clinical manifestations of hyperprolactinemia are anxiety and depression. The relationship between the response to ADs and PRL levels has been studied by Malone et al. [19]. In all examined groups, i.e., patients with major depression after electroconvulsive therapy, pharmacotherapy and psychotherapy, a high indicator PRF (prolactin response to fenfluramine) predicted a good response to AD treatment. These data suggest that the PRF may predict the response to different forms of treatment. Similarly, it has been shown that baseline cortisol, prolactin and L-tryptophan (TRP) availability may affect PRF and also influence the response to AD treatment [22]. Moreover, Depue et al. [6] reported a decrease in PRL levels in seasonal affective disorder. It is possible that low PRL levels may represent increased dopaminergic turn-

Tab. 1. Summary of antidepressant drugs that induce hyperprolactinemia based on case reports of increases in prolactin levels after antidepressant drug therapy

Antidepressants inducing hyperprolactinemia

Tricyclics Amitryptyline, imipramine, desipramine, clomipramine, amoxapine

SSRI Sertraline, fluoxetine, fluvoxamine, paroxetine, venlafaxine, citalopram, escitalopram

MA0-I Pargyline, clorgyline 


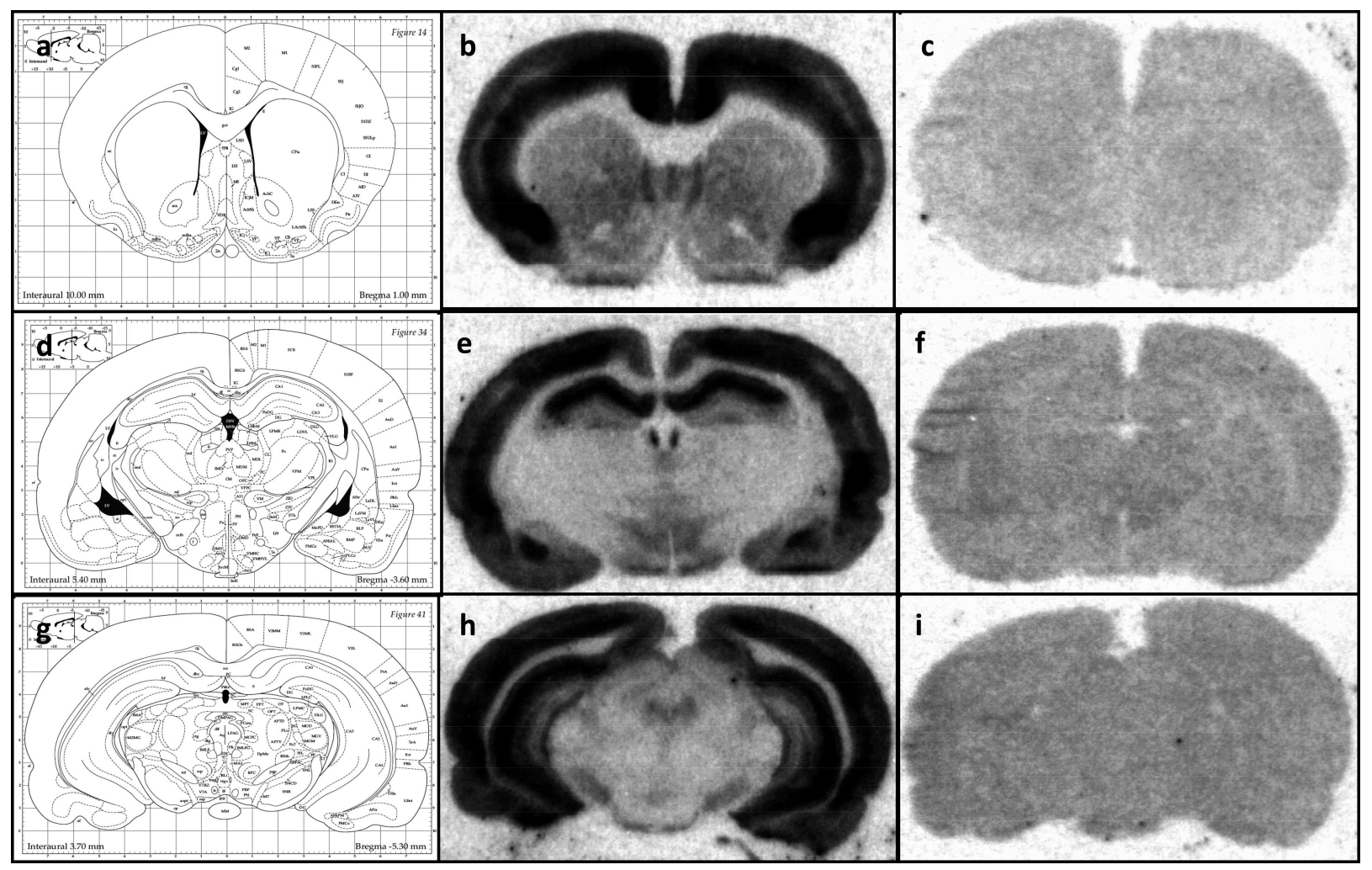

Fig. 1. Representative autoradiograms of $\left[{ }^{125}\right.$ I]SST-28 binding in rats after the CMS procedure $(\mathbf{b}, \mathbf{e}, \mathbf{h})$. Non-specific binding was determined using $1 \mu \mathrm{M} \mathrm{SST14}(\mathbf{c}, \mathbf{f}, \mathbf{j})$. The brain regions used for quantitative analysis were chosen based on the brain atlas [Paxinos and Watson: The Rat Brain in Stereotaxic Coordinates, Academic Press, London; 1998] (a, d, g)

over, which sensitizes patients to low TRP levels and makes it less likely that they will respond to treatment. However, Cleare et al. [3] showed that prolactin responses in the fenfluramine test were increased by AD treatment, but this effect was independent of whether depressed patients responded to treatment. On the other hand, response to AD treatment was associated with an increase in PRL response, while lack of response was not [18]. The mechanism of the potential link between antidepressant treatment and hyperprolactinemia remains to be fully elucidated.

PRL homeostasis is the result of a complex balance between positive and negative stimuli deriving from both the external and endogenous environments. PRL has a wide range of effects on the central nervous system (CNS), including maternal, sexual and feeding behavior, the sleep/wake cycle, the immune system, the metabolism of neurotransmitters and neuropeptides, and stress responses [1]. All of these functions of PRL are mediated by PRL receptors (PRLR). PRLR expression has been detected in various brain regions, with the highest level in the choroid plexus [27]. Peripheral and central administration of PRL have been shown to increase prolactin receptor expression in the choroid plexus [12]. The role of PRLR in the choroid plexus is to transport PRL molecules from the blood to the cerebrospinal fluid (CSF) during exposure to stress [30]. Prolactin is released into the blood from pituitary lactotroph cells in response to different stressors. Chronic restraint stress enhances the expression of the long form of the prolactin receptor ( $\mathrm{PRLR}_{\mathrm{Long}}$ ) in the choroid plexus [13]. Recently, it has been shown that PRL protects neurogenesis in the dentate gyrus of the hippocampus in chronic restraint stress [28].

All of these reports suggest the involvement of PRL in stress response. Although changes in the PRL level after acute or chronic ( 2 weeks) predictable stress (restraint or foot shock models) are well described in the literature, there is no information about changes in the level of PRL following chronic unpredictable mild stress (CMS), which is a good animal model of depression. 
CMS is currently considered one of the best animal models of depression, with high scores regarding both predictive and face validity. The CMS model was introduced by Willner and colleagues [32] based on earlier studies of the effects of ADs and stress on rat behavior. In this model, rats are subjected to mild stressors for a few weeks; each stimulus is mild, but because they are imposed on the animals every day, they cause various perturbations similar to the symptoms typically observed in the clinic, such as the inhibition of exploratory and sexual activity, the inhibition of dominant behavior, disturbances in the circadian rhythm and sleep cycle and weight gain. There are also disturbances in the functionality of the immunological and endocrine systems. The most frequently studied effect of CMS is the lower sensitivity of the stressed animals to rewarding stimuli, and this feature is regarded as a good animal model of anhedonia, one of the main symptoms of depression in humans. Anhedonia in animals has been studied with the use of various experimental paradigms, such as place preference or self-stimulation, but the most useful approach is to observe and measure the amount of $1 \%$ sucrose solution consumed by the animals, which is significantly decreased in animals subjected to CMS. Repeated administration of ADs to these animals restores the normal consumption of sucrose. All classes of ADs have been shown to be effective in this model, including tricyclic ADs, selective serotonin reuptake inhibitors, monoaminooxidase inhibitors and atypical ADs. Similarly, non-pharmacological treatments also have been shown to be effective in this model, including deprivation of the REM phase of sleep or electroconvulsive shock. These results speak in favor of the high predictive validity of the model [31]. The effects of antidepressant treatment in the CMS model occur progressively and are significant after 3-4 weeks of drug administration, which is consistent with the clinical situation. Moreover, similar to the clinic, some of the animals subjected to CMS are resistant to both stress and antidepressants. Therefore, we decided to use the CMS paradigm, which allows correlations among the behavioral response to stress, antidepressant treatment and PRL levels to be studied. From the obtained results, it can be concluded that 2 weeks of the CMS procedure is sufficient to select the animals that are reactive to stress (stress-R), i.e., displaying anhedonia, and the animals that are stress-non reactive (stress-NR) as far as behavioral measures are concerned. However, this stress-NR group differs from the stress- $\mathrm{R}$ one in reactivity at the biochemical level; in this group, an elevated level of PRL in plasma, a decrease in dopamine (DA) release in the tuberoinfundibular circuit, an increase in $\left[{ }^{125} \mathrm{I}\right] \mathrm{PRL}$ binding to PRL receptors in the choroid plexus, an increase in the mRNA encoding long form of PRL receptors in the arcuate nucleus, a decrease in the mRNA encoding its short form, and a decrease in the mRNA encoding the dopamine $D_{2}$ receptor $\left(D_{2} R\right)$ were observed. All of these alterations indicate that this group of animals are perhaps resilient to stress not "resistant" - and the indicated parameters are certainly involved in the phenomenon of stress resilience.

The continuation of the CMS procedure for an additional 5 weeks shows - through the lack of any statistically significant changes in the measured biochemical parameters - habituation to the stressful conditions. However, the most interesting result was the upregulation of PRL receptors in the choroid plexus of rats subjected to the full CMS procedure combined with treatment with IMI, which may support the role of this receptor in the mechanisms of antidepressant action.

Physiological control of PRL secretion is primarily exerted by the inhibiting action of DA. DA, secreted in the hypothalamic periventricular zone (periventricular nucleus and arcuate nucleus) and released from neuronal projections in the median eminence, reaches the anterior pituitary gland through portal vessels (TIDA tuberoinfundibular dopamine pathway). The DA-mediated inhibition of PRL secretion occurs via the binding of $\mathrm{D}_{2}$ receptors on the membrane of lactotroph cells and involves several signal transduction systems, resulting in the inhibition of PRL gene transcription and the reduction of PRL synthesis and release [1]. In addition to the inhibitory action of DA, there are other PRL-inhibiting factors in the CNS, including $\gamma$-aminobutyric acid (GABA), acetylcholine, norepinephrine and somatostatin (SST) [16].

\section{Somatostatin}

In major depression, in addition to the levels of classical neurotransmitters, neuropeptide levels are also altered in certain brain regions. One of these neuropep- 
tides is SST, which acts as both a hormone and a neurotransmitter. It has two active forms (SST-14 and SST-28) that are derived from the same precursor [9]. The SST inhibiting factor was initially discovered as a neurohormone that inhibits growth hormone $(\mathrm{GH})$ secretion from anterior pituitary somatotroph cells. This function is performed in hypophysiotropic neurons located in the anterior periventricular hypothalamic nucleus, which projects to the median eminence and releases the peptide in the capillaries of the hypothalamo-hypophyseal portal vessels, thus directly connecting the brain to the anterior pituitary. SST is also a potent inhibitor of many hormonal secretions, including PRL. SST receptors $\left(\mathrm{sst}_{1} \mathrm{R}-\mathrm{sst}_{5} \mathrm{R}\right)$ are Gprotein coupled receptors responsible for the inhibition of adenylate cyclase, activation of potassium channels and stimulation of tyrosine phosphatase. SST is widely distributed within the CNS, as presented in Figure 1.

There is evidence that SST may be involved in emotional processes such as anxiety and depression. Intracerebral administration of SST to animals induces anxiolytic and antidepressant-like effects in behavioral tests [9]. Moreover, the effects of $\mathrm{sst}_{2} \mathrm{R}$ and/or sst $_{3} \mathrm{R}$ receptor agonists in the elevated plusmaze and forced swim tests were equivalent to the effects of anxiolytic and antidepressant drugs, which indicates the potential role of these receptor subtypes in the action of antidepressants. The antidepressant effect may result from positive SST effects on serotonin release, while the anxiolytic-like effects of SST seem to result from the general inhibitory effects of this neuropeptide [20]. Although results obtained by Tripp et al. suggest an impaired excitation/inhibition balance in Major Depression Disorder (MDD) potentially mediated by decreased GABA content, recently obtained results indicate downregulation of the SST levels in the anterior cingulate cortex, dorsolateral prefrontal cortex and amygdala [15, 25, 29] of MDD patients. SST is coexpressed and coreleased with GABA in the interneurons in various regions of the human brain, including the anterior cingulated cortex. Thus, lower levels of SST (downregulated by $\sim 30 \%$ at the mRNA level and $\sim 20 \%$ at the SST precursor level) may provide a starting point to further characterize the effects of its changes on MDD [29]. It has also been reported that the level of SST is decreased in the cerebrospinal fluid (CSF) of depressed patients [11]. Chronic administration of ADs influences the SST levels and its receptors in the rat brain [21]. Re- cently, it has been shown that imipramine upregulates SST release in the mouse hypothalamus [20].

Moreover, SST expression has also been shown to be positively regulated by brain-derived neurotrophic factor (BDNF). Recent studies using transgenic mice models suggest a complex mechanism of low constitutive and activity-dependent BDNF function, particularly affecting SST/NPY-expressing GABA neurons in the amygdala [15]. SST was also investigated in the context of its influence on sleep quality as a potential therapeutic approach to sleep disturbances in MDD, although the results were inconsistent.

Despite these reports, very interesting results have been reported recently, indicating that populations of interneurons in the adult rat hypothalamus switch between DA and SST expression in response to exposure to short- and long-day photoperiods [7]. The shifts in SST/DA expression are regulated at the transcriptional level, are matched by parallel changes in postynaptic $\mathrm{D}_{2} \mathrm{R} / \mathrm{sst}_{2} \mathrm{R} / \mathrm{sst}_{4} \mathrm{R}$ expression, and have profound effects on behavior. Increased DA signaling during short days results in the decreased release of CRF from target neurons and consequently leads to a decrease in CRF and corticosteroids in the plasma and a decreased number of SST cells. These conditions are associated with a decrease in stress behaviors in nocturnal rodents. The converse is observed with decreased DA signaling during long-day conditions. These data indicate the role of SST and DA in at least some depressive disorders, e.g., seasonal depression.

The results obtained in our recent experiments using the CMS model indicate that the levels of SST receptors are influenced both by chronic stress and antidepressant treatment. Interestingly, in the cingulate and primary motor cortexes, we observed a statistically significant decrease in SST binding sites in the group of animals that are non-responsive to imipramine (IMI) treatment compared to rats that respond to this drug. Because some studies have indicated that IMI increases the level of SST in the cingulate cortex, the reduction of SST receptors in the rats subjected to CMS that do not respond to IMI may indicate its involvement in the mechanisms of drug resistance of these animals.

SST receptors are mainly localized in the brain cortex, striatum and limbic system, where they colocalize with DA receptors, e.g., $s_{2} \mathrm{R} / \mathrm{sst}_{4} \mathrm{R}$ colocalizes with $\mathrm{D}_{2} \mathrm{R}$ on CRF neurons in the rat hypothalamus [7] or $\mathrm{D}_{2} \mathrm{R}$ and $\mathrm{sst}_{5} \mathrm{R}$ colocalize in the striatum and pyrami- 
dal neurons in the cerebral cortex [23]. It has also been shown that SST receptors may form functional hetero-oligomers with $\mathrm{D}_{2}$ receptors [8]. Recently, data were reported that indicated the role of heterooligomerization of SST receptors with $\mathrm{D}_{2}$ receptors in hyperprolactinemia and pituitary tumors [10]. It has also been shown that the chimeric SST-DA compound BIM-23A760 suppressed PRL secretion in DAresistant prolactinomas [14]. Direct interaction between dopaminergic and somatostatinergic receptors has been recently shown using an in vitro system and biophysical approach. Additionally, it has been found that antidepressant drugs promote the heterodimerization of $\mathrm{D}_{2}$ and sst ${ }_{5}$ receptors [26].

\section{Conclusion}

Considering these data, it seems that it would be very interesting to study the correlation of these "opposing" neurohormones, SST and PRL, in animals subjected to CMS, particularly responders and nonresponders to stress and the administration of antidepressant drugs, as these two neurohormones might play an important role in the mechanism of stress resilience as well as the mechanism of action of antidepressant drugs.

\section{Acknowledgment:}

Supported by grant DeMeTer (project

number:POIG.01.01.02-12-004/09; 3.6.) and statutory activity of Institute of Pharmacology Polish Academy of Sciences.

\section{References:}

1. Ben-Jonathan N, LaPensee CR, LaPensee EW: What can we learn from rodents about prolactin in humans? Endocr Rev, 2008, 29, 1-41.

2. Brady LS, Gold PW, Herkenham M, Lynn AB, Whitfield HJ Jr: The antidepressants fluoxetine, idazoxan and phenelzine alter corticotropin-releasing hormone and tyrosine hydroxylase mRNA levels in rat brain: therapeutic implications. Brain Res, 1992, 572, 117-125.

3. Cleare AJ, Murray RM, O'Keane V: Assessment of serotonergic function in major depression using $d$-fenfluramine: relation to clinical variables and antidepressant response. Biol Psychiatry, 1998, 44, 555-561.
4. Coker F, Taylor D: Antidepressant-induced hyperprolactinaemia: incidence, mechanisms and management. CNS Drugs, 2010, 24, 563-574.

5. Deecher D, Andree TH, Sloan D, Schechter LE: From menarche to menopause: exploring the underlying biology of depression in women experiencing hormonal changes. Psychoneuroendocrinology, 2008, 33, 3-17.

6. Depue RA, Arbisi P, Krauss S, Iacono WG, Leon A, Muir R, Allen J: Seasonal independence of low prolactin concentration and high spontaneous eye blink rates in unipolar and bipolar II seasonal affective disorder. Arch Gen Psychiatry, 1990, 47, 356-364.

7. Dulcis D, Jamshidi P, Leutgeb S, Spitzer NC: Neurotransmitter switching in the adult brain regulates behavior. Science, 2013, 340, 449-453.

8. Durán-Prado M, Malagón MM, Gracia-Navarro F, Castaño JP: Dimerization of G protein-coupled receptors: new avenues for somatostatin receptor signalling, control and functioning. Mol Cell Endocrinol, 2008, 286, 63-68.

9. Engin E, Treit D: Anxiolytic and antidepressant actions of somatostatin: the role of sst 2 and sst 3 receptors. Psychopharmacology, 2009, 206, 281-289.

10. Ferone D, Gatto F, Arvigo M, Resmini E, Boschetti M, Teti C, Esposito D, Minuto F: The clinical-molecular interface of somatostatin, dopamine and their receptors in pituitary pathophysiology. J Mol Endocrinol, 2009, 42, 361-370.

11. Frye MA, Pazzaglia PJ, George MS, Luckenbaugh DA, Vanderham E, Davis CL, Rubinow DR, Post RM: Low CSF somatostatin associated with response to nimodipine in patents with affective illness. Biol Psychiatry, 2003, 53, 180-183.

12. Fujikawa T, Soya H, Tamashiro KL, Sakai RR, McEwen BS, Nakai N, Ogata M et al.: Prolactin prevents acute stress-induced hypocalcemia and ulcerogenesis by acting in the brain of rat. Endocrinology, 2004, 145, 2006-2013.

13. Fujikawa T, Soya H, Yoshizato H, Sakaguchi K, Doh-Ura K, Tanaka M, Nakashima K: Restraint stress enhances the gene expression of prolactin receptor long form at the choroid plexus. Endocrinology, 1995, 136, 5608-5613.

14. Fusco A, Gunz G, Jaquet P, Dufour H, Germanetti AL, Culler MD, Barlier A, Saveanu A: Somatostatinergic ligands in dopamine-sensitive and -resistant prolactinomas. Eur J Endocrinol, 2008, 158, 595-603.

15. Guilloux JP, Douillard-Guilloux G, Kota R, Wang X, Gardier AM, Martinowich K, Tseng GC et al.: Molecular evidence for BDNF- and GABA-related dysfunctions in the amygdala of female subjects with major depression. Mol Psychiatry, 2012, 17, 1130-1142.

16. Ignacak A, Kasztelnik M, Sliwa T, Korbut RA, Rajda K, Guzik TJ: Prolactin-not only lactotrophin. A "new" view of the "old" hormone. J Physiol Pharmacol, 2012, 63, 435-443. Review.

17. Kessler RC: Epidemiology of women and depression. Affect Disord, 2003, 74, 5-13.

18. Leatherman ME, Ekstrom RD, Corrigan M, Carson SW, Mason G, Golden RN: Central serotonergic changes fol- 
lowing antidepressant treatment: a neuroendocrine assessment. Psychopharmacol Bull, 1993, 29, 149-154.

19. Malone KM, Thase ME, Mieczkowski T, Myers JE, Stull SD, Cooper TB, Mann JJ: Fenfluramine challenge test as a predictor of outcome in major depression. Psychopharmacol Bull, 1993, 29, 155-161.

20. Nilsson A, Stroth N, Zhang X, Qi H, Fälth M, Sköld K, Hoyer D et al.: Neuropeptidomics of mouse hypothalamus after imipramine treatment reveal somatostatin as a potential mediator of antidepressant effects. Neuropharmacology, 2012, 62, 347-357.

21. Pallis E, Vasilaki A, Fehlmann D, Kastellakis A, Hoyer D, Spyraki C, Thermos K: Antidepressants influence somatostatin levels and receptor pharmacology in brain. Neuropsychopharmacology, 2009, 34, 952-963.

22. Porter RJ, Mulder RT, Joyce PR: Baseline prolactin and L-tryptophan availability predict response to antidepressant treatment in major depression. Psychopharmacology, 2003, 165, 216-221.

23. Rocheville M, Lange DC, Kumar U, Patel SC, Patel RC, Patel YC: Receptors for dopamine and somatostatin: formation of hetero-oligomers with enhanced functional activity. Science, 2000, 288, 154-157.

24. Rush AJ, Trivedi MH, Wisniewski SR, Nierenberg AA, Stewart JW, Warden D, Niederehe G et al.: Acute and longer-term outcomes in depressed outpatients requiring one or several treatment steps: a STAR*D report. Am J Psychiatry, 2006, 163, 1905-1917.

25. Sibille E, Morris HM, Kota RS, Lewis DA: GABArelated transcripts in the dorsolateral prefrontal cortex in mood disorders. Int J Neuropsychopharmacol, 2011, 14, 721-734.
26. Szafran K, Lukasiewicz S, Faron-Górecka A, Kolasa M, Kuśmider M, Solich J, Dziedzicka-Wasylewska M: Antidepressant drugs promote the heterodimerization of the dopamine D2 and somatostatin Sst5 receptors-

fluorescence in vitro studies. Pharmacol Rep, 2012, 64, $1253-1258$.

27. Tabata H, Kobayashi M, Ikeda JH, Nakao N, Saito TR, Tanaka M: Characterization of multiple first exons in murine prolactin receptor gene and the effect of prolactin on their expression in the choroid plexus. J Mol Endocrinol, 2012, 48, 169-176.

28. Torner L, Karg S, Blume A, Kandasamy M, Kuhn HG, Winkler J, Aigner L, Neumann ID: Prolactin prevents chronic stress-induced decrease of adult hippocampal neurogenesis and promotes neuronal fate. J Neurosci, 2009, 29, 1826-1833.

29. Tripp A, Kota RS, Lewis DA, Sibille E: Reduced somatostatin in subgenual anterior cingulate cortex in major depression. Neurobiol Dis, 2011, 42, 116-124.

30. Walsh RJ, Slaby FJ, Posner BI: A receptor-mediated mechanism for the transport of prolactin from blood to cerebrospinal fluid. Endocrinology, 1987, 120, 1846-1850.

31. Willner P: Chronic mild stress (CMS) revisited: consistency and behavioural-neurobiological concordance in the effects of CMS. Neuropsychobiology, 2005, 52, 90-110.

32. Willner P: Validity, reliability and utility of the chronic mild stress model of depression: a 10-year review and evaluation. Psychopharmacology, 1997, 134, 319-329.

Received: July 26, 2013; in the revised form: September 11, 2013; accepted: September 13, 2013. 